\title{
THE AGE OF THE DIRECTLY IMAGED PLANET HOST STAR $\kappa$ ANDROMEDAE DETERMINED FROM INTERFEROMETRIC OBSERVATIONS
}

\author{
Jeremy Jones $^{1}$, R. J. White ${ }^{1}$, S. Quinn ${ }^{1}$, M. Ireland ${ }^{2}$, T. Boyajian ${ }^{3}$, G. Schaefer ${ }^{1}$, and E. K. Baines ${ }^{4}$ \\ ${ }^{1}$ Center for High Angular Resolution Astronomy and Department of Physics and Astronomy, Georgia State University, \\ 25 Park Place, Suite 605, Atlanta, GA 30303, USA; jones@astro.gsu.edu \\ ${ }^{2}$ Research School of Astronomy \& Astrophysics, Australian National University, Canberra, ACT 2611, Australia \\ ${ }^{3}$ Department of Astronomy, Yale University, New Haven, CT 06511, USA \\ ${ }^{4}$ Remote Sensing Division, Naval Research Laboratory, 4555 Overlook Avenue SW, Washington, DC 20375, USA \\ Received 2016 February 8; revised 2016 March 25; accepted 2016 April 4; published 2016 April 22
}

\begin{abstract}
$\kappa$ Andromedae, an early-type star that hosts a directly imaged low-mass companion, is expected to be oblate due to its rapid rotational velocity $\left(v \sin i=\sim 162 \mathrm{~km} \mathrm{~s}^{-1}\right)$. We observed the star with the CHARA Array's optical beam combiner, PAVO, measuring its size at multiple orientations and determining its oblateness. The interferometric measurements, combined with photometry and this $v \sin i$ value are used to constrain an oblate star model that yields the fundamental properties of the star and finds a rotation speed that is $\sim 85 \%$ of the critical rate and a low inclination of $\sim 30^{\circ}$. Three modeled properties (the average radius, bolometric luminosity, and equatorial velocity) are compared to MESA evolution models to determine an age and mass for the star. In doing so, we determine an age for the system of $47_{-40}^{+27} \mathrm{Myr}$. Based on this age and previous measurements of the companion's temperature, the BHAC15 evolution models imply a mass for the companion of $22_{-9}^{+8} M_{\mathrm{J}}$.
\end{abstract}

Key words: planetary systems - stars: early-type - stars: evolution - stars: individual (kappa Andromedae) - stars: rotation - techniques: interferometric

\section{INTRODUCTION}

The vast majority of exoplanets have been discovered with indirect methods such as studying the radial velocity variations induced on the host star or measuring how much light from the host star is blocked by the transiting planet (Winn \& Fabrycky 2015). However, the spectral lines of typical earlytype stars are rotationally broadened, making them not conducive to the precise radial velocity measurements necessary for planetary detection and confirmation. In fact, only 15 substellar mass companions have been discovered around early-type stars (Hartman et al. 2015 and references therein). Five of these were discovered using the transit method and the remaining 10 were discovered with direct imaging. Accurate age estimates of stars that harbor directly imaged companions are necessary to determine the masses of the companions because these masses are all dependent on evolution models designed for low-mass objects that cool with age (e.g., Baraffe et al. 2003).

The B9IVn star, $\kappa$ Andromedae A (hereafter, $\kappa$ And A; other identifiers include 19 And, HD 222439, HIP 116805, HR 8976, and Téng Shé èrshíy - The Twenty First Star of Flying Serpent) is the hottest $\left(T_{\text {eff }} \sim 11,200 \mathrm{~K}\right)$ and most massive ( $M$ $\sim 2.8 M_{\odot}$ ) star known to host a directly imaged companion (hereafter, $\kappa$ And b), discovered by Carson et al. (2013). The host star is rapidly rotating with a $v \sin i$ of $\sim 160 \mathrm{~km} \mathrm{~s}^{-1}$ (Glebocki \& Gnacinski 2005; Royer et al. 2007) and is at a distance of $51.6 \pm 0.5 \mathrm{pc}$ (van Leeuwen 2007). Zuckerman et al. (2011) consider it to be a member of the 30 Myr Columba association. Carson et al. (2013) adopted this age for $\kappa$ And A and used DUSTY cooling models (Baraffe et al. 2003) to determine the mass of $\kappa$ And $\mathrm{b}$ to be $12.8_{-1.0}^{+2.0} M_{\text {Jup }}$. Hinkley et al. (2013, hereafter H13) estimated the age of the system to be $220 \pm 100 \mathrm{Myr}, \sim 7$ times older than the age of Columba by comparing $\log (g)$ and $T_{\text {eff }}$ estimates to the predictions of stellar models. At this age, the mass of $\kappa$ And $\mathrm{b}$ would be $50_{-13}^{+16} M_{\text {Jup }}$, much larger than the traditional boundary of $\sim 13 \mathrm{M}_{\text {Jup }}$ between planets and brown dwarfs (Spiegel et al. 2011; Mollière and Mordasini 2012; Bodenheimer et al. 2013).

Other studies estimate a range of ages for $\kappa$ And A. Bonnefoy et al. (2014) compare the star's position on an $M_{V}$ versus $B-V$ color-magnitude diagram to the predictions of the Ekström et al. (2012) evolution models and find an age $\lesssim 250$ Myr. David \& Hillenbrand (2015, hereafter DH15) use high-precision $u v b y \beta$ photometry to estimate the $T_{\text {eff }}$ and $\log (g)$ of a large sample of early-type stars, including $\kappa$ And A, and estimate ages by comparing these values to the predictions of the evolution models of Bressan et al. (2012) and Ekström et al. (2012). With their Bayesian analysis, they find a $95 \%$ confidence interval of 29-237 Myr for $\kappa$ And A and argue that it is not coeval with Columba. Alternatively, the Bayesian analysis of Brandt \& Huang (2015) suggests that coevality with Columba cannot be ruled out.

To more accurately determine the properties of $\kappa$ And A, including its age, we present interferometric observations of $\kappa$ And A taken with the PAVO beam combiner on the CHARA Array. Using the model described in Jones et al. (2015, hereafter J15), we determine various fundamental parameters of $\kappa$ And $\mathrm{A}$, including its radius, temperature, inclination, and luminosity, and based on comparisons with the MESA evolution model (Paxton et al. 2011, 2013), determine its mass and age. This procedure was validated using coeval members of the Ursa Major Moving Group (UMMG), showing that the MESA evolution models are appropriate for dating rapidly rotating stars by finding coeval ages between rapidly and slowly rotating members of the UMMG and by estimating an age for the group in agreement with the admittedly large range of age estimates for the group. With an age for the $\kappa$ And system, we estimate a mass for the companion by using the BHAC15 evolution models (Baraffe et al. 2015). 
Table 1

Observing Log

\begin{tabular}{|c|c|c|c|c|c|}
\hline Cal HD & Cal Diameter (mas) & Baseline & \# Observations & \# Visibilities & Date \\
\hline 222304 & $0.263 \pm 0.026$ & S2-E2 & 4 & 92 & 2012 Dec 21 \\
\hline 220885 & $0.230 \pm 0.023$ & S2-E2 & 4 & 92 & 2012 Dec 21 \\
\hline 222304 & $0.263 \pm 0.026$ & W1-E1 & 1 & 23 & 2013 Aug 2 \\
\hline 220885 & $0.230 \pm 0.023$ & S1-E1 & 2 & 46 & 2013 Aug 2 \\
\hline 220885 & $0.230 \pm 0.023$ & S1-E1 & 3 & 69 & 2013 Aug 3 \\
\hline 220885 & $0.230 \pm 0.023$ & W1-S1 & 3 & 69 & 2013 Aug 3 \\
\hline 222304 & $0.263 \pm 0.026$ & W1-S1 & 3 & 69 & 2013 Aug 3 \\
\hline 220885 & $0.230 \pm 0.023$ & E1-W2 & 4 & 92 & 2013 Aug 5 \\
\hline
\end{tabular}

\section{OBSERVATIONS AND DATA REDUCTION}

\subsection{Visibilities}

Observations of $\kappa$ And A were made using the Precision Astronomical Visible Observations (PAVO) beam combiner on the Center for High Angular Resolution Astronomy (CHARA) Array (Ireland et al. 2008; ten Brummelaar et al. 2005). The CHARA Array is an optical interferometer made up of six 1-m telescopes arranged in a Y-shaped configuration with a maximum baseline of $331 \mathrm{~m}$. Each telescope is named with a letter designating its arm ("S"- - south, "E"-east, "W"-west) and a number designating its place on the arm ("1"- - outer, "2"-inner). PAVO was used in its two-telescope mode and produces 23 spectrally dispersed squared-visibility measurements for each observation over a wavelength range of $0.65-0.79 \mu \mathrm{m}$. In total, we made 24 observations yielding 552 spectrally dispersed squared-visibility measurements over four nights using five different baselines in order to measure its oblateness.

We observe two different calibrator stars (HD 222304 and HD 220885) shortly before and after (within $\sim 30$ minutes) our observations of $\kappa$ And A and by doing so, we can account for how the atmosphere dampens the measured visibilities of the target star (Roddier 1981p. 281; Boden 2007). We predict that these calibrator stars have small angular diameters $(<0.27$ mas $)$ based on fitting photometric energy distributions to measured photometry. We reduce and calibrate the data with the reduction pipeline of Ireland et al. (2008). Table 1 lists the dates observations were made, how many observations were made, the baselines used, and the calibrator used.

\subsection{Photometry}

We take advantage of the ample photometric observations of $\kappa$ And A that have been made over the years, using photometry from the following sources: Johnson $U B V$ from Mermilliod (2006); Strömgren uvby from Hauck \& Mermilliod (1997); Johnson $J K$ from Selby et al. (1988); and UV photometry with wavelengths ranging from 1500 to $3300 \AA$ from Thompson et al. (1978) and Wesselius et al. (1982). IUE spectrophotometry (Boggess et al. 1978) exists for $\kappa$ And A that we do not use, but matches to our model spectral energy distribution (SED) and the broadband UV photometry that we use. Following arguments from J15, we adopt an uncertainty of $0.03 \mathrm{mag}$ for all photometric values.

\section{MODELING OF STELLAR PROPERTIES}

\subsection{Oblate Star Model}

Because of $\kappa$ And A's rapid rotation $(v \sin i=161.6 \pm$ $22.2 \mathrm{~km} \mathrm{~s}^{-1}$; Glebocki \& Gnacinski 2005; Royer et al. 2007), the limb-darkened disk traditionally used to model interferometric data is insufficient. Rapid rotation causes a star to have a radius at the equator larger than its radius at the pole. The ratio between the equatorial and polar radii can be as high as 1.5 when the star is rotating at its breakup velocity (van Belle 2012). The thicker equatorial bulge of a rapid rotator results in the equator being both cooler and fainter than the pole. This effect, known as gravity darkening, is correlated with the local surface gravity (von Zeipel 1924a, 1924b).

We account for both the oblateness and gravity darkening of $\kappa$ And A by using the model of J15, which compares observed photometry and interferometric visibilities to values generated by a model star that incorporates the effects of solid-body rotation, known as a "Roche model" (van Belle 2012; Roche 1873). The model photometry is calculated by integrating ATLAS model SEDs (Castelli \& Kurucz 2004) over the visible surface of the star, convolving the integrated SED with the appropriate filter bandpasses, and converting the resulting fluxes into magnitudes. To calculate model visibilities, we generate an image of the model at the observed bandpasses. The model visibilities are calculated by taking the Fourier transform of this image and sampling the transform at the observed spatial frequencies.

The model and parameters calculated by the model are described in detail in J15, but we note three slight differences here. One such difference is that we use ATLAS model SEDs for this work rather than the PHOENIX model SEDs used in J15 (Husser et al. 2013), since they extend to effective temperatures hotter than $12,000 \mathrm{~K}$. Another difference is that we only use the gravity-darkening law of Espinosa Lara \& Rieutord (2011) because the data are not sensitive to differences in gravity-darkening laws and this law is supported by previous interferometric observations.

The final difference is in how uncertainties are calculated. Under the assumption that the uncertainties in the free parameters are Gaussian and that the model parameters are linear, we use the following prescription to determine uncertainties in the free parameters: because the $\chi^{2}$ values determined by the models are larger than 1 , for each data set (photometry and visibilities), we scale the $\chi^{2}$ (both reduced and unreduced) such that the reduced $\chi^{2}$ is 1 . The free parameters are then varied individually until the scaled, unreduced $\chi^{2}$ increases by 1 . This 
gives two sets of uncertainties for the free parameters-one for the photometry and one for the visibilities, with the exception of the position angle, which is only probed by the visibilities. The final uncertainty in each free parameter is determined by adding the two uncertainties in quadrature under the assumption that the visibilities and photometry are independent. The uncertainty in the position angle is determined only by comparison with the visibilities. These uncertainties are then propagated to determine the uncertainties in the derived parameters. We caution the reader that these uncertainties are statistical and do not account for systematic uncertainties such as errors in the model spectra, gravity-darkening law, etc. The coevality of oblate and nonoblate A-stars in the UMMG, determined using this model (J15), suggests that these systematic uncertainties do not dominate the errors.

Figure 1 illustrates the best-fitting model by showing the modeled visibilities and photometry as well as the modeled photosphere overlaid with approximate radius measurements at various orientations. Using four different metallicities (justified below), the best-fit modeled properties are listed in rows 3-7 of Table 2, and the properties derived from these are in rows $8-20$ of Table 2.

\subsection{Stellar Evolution Models}

We take the average radius $\left(R_{\text {avg }}\right)$, total bolometric luminosity $\left(L_{\mathrm{bol}}\right)$, and equatorial rotation velocity $\left(V_{\mathrm{e}}\right)$ shown in Table 2 and use MESA evolution models (Paxton et al. 2011, 2013) to determine the age and mass of $\kappa$ And A by comparing the modeled values to MESA's predictions for given masses, ages, and initial rotation rates. MESA models are used because they can account for the rapid rotation of $\kappa$ And A. The uncertainties in the mass and age are based on propagated uncertainties in stellar properties (J15).

One systematic source of uncertainty that is difficult to account for in this analysis is the metallicity of the evolution model. There are several reasons to suspect that the subsolar surface abundance of $\kappa$ And A (e.g., $[\mathrm{M} / \mathrm{H}]=-0.32 \pm 0.15$; Wu et al. 2011) does not trace its internal abundance. First, the surface abundances of A- and B-stars within populations believed to be chemically homogeneous span a broad range. Moreover, there is evidence that photospheric abundances are anti-correlated with projected rotational velocity $(v \sin i)$, becoming distinctively subsolar (e.g., $\lesssim-0.30$ ) when projected rotational velocities exceed $\sim 150 \mathrm{~km} / \mathrm{s}$ (e.g., Takeda \& Sadakane 1997; Varenne \& Monier 1999). Thus, there is reason to suspect that the internal abundance of $\kappa$ And A is more metalrich than is observed in its photosphere. Finally, as emphasized by H13, the Galaxy has not recently produced many stars that are this metal-poor. To quantify this, we consider the sample of open clusters with metallicity measurements assembled in Chen et al. (2003). These 77 clusters have a mean metallicity of $0.00 \mathrm{dex}$ and a standard deviation of $0.14 \mathrm{dex}$; the most metal-poor cluster among them has a metallicty of -0.34 dex. Given these considerations, we adopt a solar metallicity $([\mathrm{M} / \mathrm{H}]=0.00 \mathrm{dex}$, $Z=0.0153$, Caffau et al. 2011) for $\kappa$ And A, with an uncertainty of 0.14 dex. Nevertheless, we also consider a metallicity of $[\mathrm{M} / \mathrm{H}]=-0.28$ dex as a $2 \sigma$ extremum in our analysis. Figure 2 shows the average radius and temperature of $\kappa$ And A overlaid with mass tracks and isochrones from the MESA evolution models for solar metallicity that have been interpolated to the modeled rotational velocity.

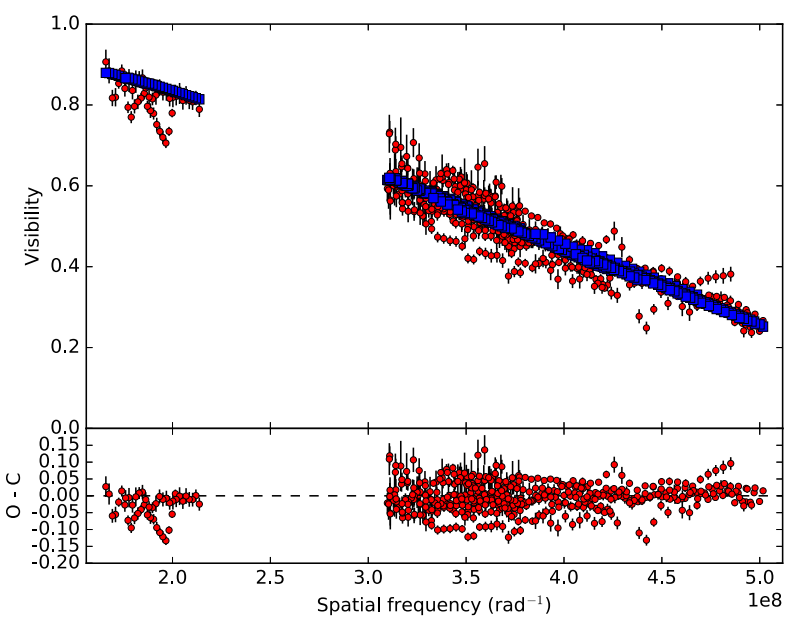

(a)

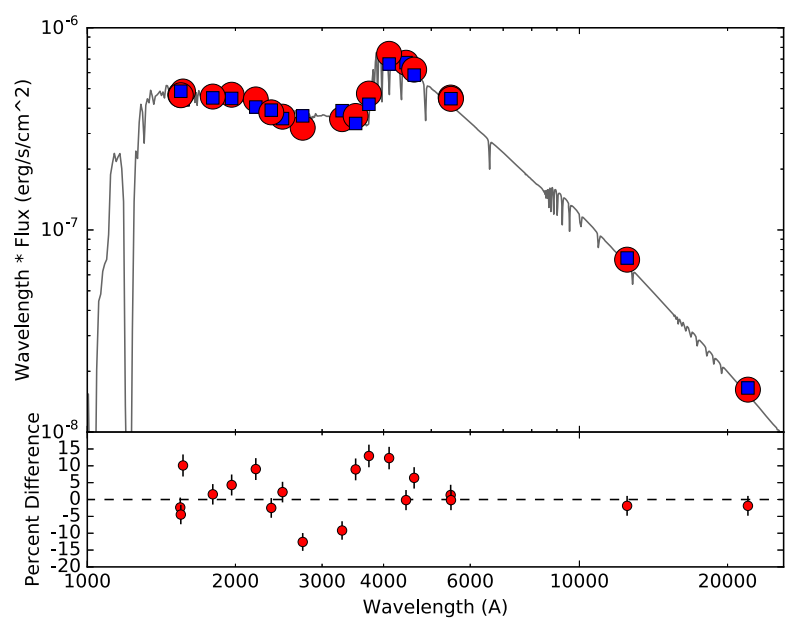

(b)

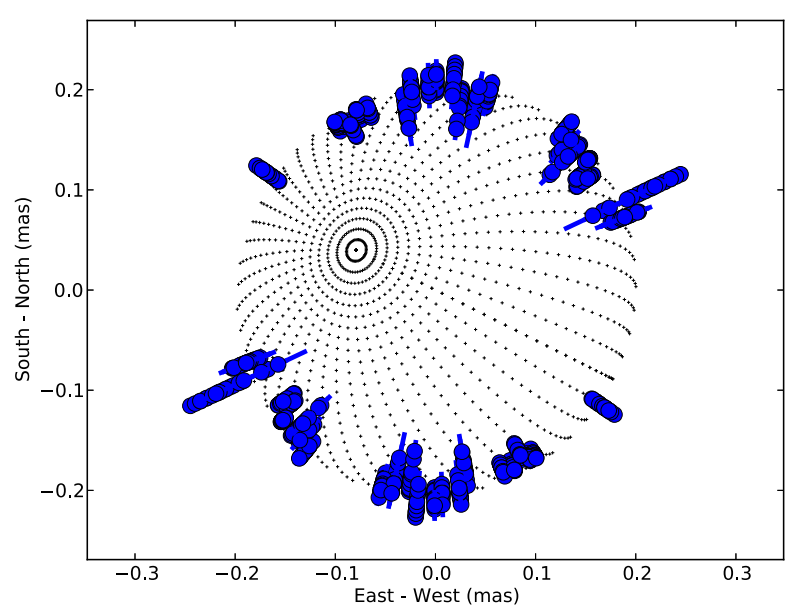

(c)

Figure 1. Top: observed (red circles) and best-fit model visibilities (blue squares) vs. spatial frequencies for the solar metallicity model. Middle: observed (red circles) and best-fit model (blue squares) photometric fluxes versus wavelength for the solar metallicity model. The modeled SED is shown in gray. Bottom: the photosphere of the best-fitting model of $\kappa$ And A. The black points represent a grid of colatitudes and longitudes on the near side of the model. The blue circles represent a radius fitted to each individual visibility at the appropriate baseline orientation observed. The data are duplicated at $180^{\circ}$ orientation. 
Table 2

Model Results

\begin{tabular}{|c|c|c|c|c|}
\hline \multicolumn{5}{|c|}{ Properties of $\kappa$ And A } \\
\hline Internal $[\mathrm{M} / \mathrm{H}]$ & +0.14 & $0.00^{\mathrm{a}}$ & -0.14 & -0.28 \\
\hline Internal $Z$ & 0.0211 & 0.0153 & 0.0111 & 0.0080 \\
\hline Equatorial Radius, $R_{\mathrm{e}}\left(R_{\odot}\right)$ & $2.331_{-0.011}^{+0.068}$ & $2.303_{-0.016}^{+0.039}$ & $2.326_{-0.023}^{+0.029}$ & $2.366_{-0.027}^{+0.023}$ \\
\hline Equatorial Velocity, $V_{\mathrm{e}}\left(\mathrm{km} \mathrm{s}^{-1}\right)$ & $354.8_{-35.4}^{+7.3}$ & $283.8_{-16.1}^{+13.4}$ & $322.5_{-13.3}^{+19.2}$ & $376.6_{-11.5}^{+14.4}$ \\
\hline Polar Position Angle, $\psi\left({ }^{\circ}\right)$ & $69.6_{-0.9}^{+3.2}$ & $63.4_{-1.0}^{+5.2}$ & $69.3_{-2.7}^{+0.5}$ & $72.8_{-1.2}^{+0.9}$ \\
\hline \multicolumn{5}{|c|}{ Properties Derived from Oblate Star Model } \\
\hline Gravity Darkening, $\beta$ & $0.181_{-0.002}^{+0.011}$ & $0.202_{-0.004}^{+0.004}$ & $0.188_{-0.006}^{+0.004}$ & $0.166_{-0.006}^{+0.004}$ \\
\hline Angular Rotation Rate, $\omega$ & $0.947_{-0.039}^{+0.007}$ & $0.854_{-0.028}^{+0.021}$ & $0.921_{-0.017}^{+0.021}$ & $0.978_{-0.008}^{+0.008}$ \\
\hline Average Temperature, $T_{\text {avg }}(\mathrm{K})^{\mathrm{b}}$ & $11250_{-163}^{+133}$ & $11327_{-44}^{+421}$ & $11290_{-53}^{+291}$ & $11307_{-295}^{+43}$ \\
\hline Polar Surface Gravity, $\log \left(g_{\mathrm{p}}\right)(\mathrm{cgs})$ & $4.373_{-0.036}^{+0.008}$ & $4.296_{-0.012}^{+0.019}$ & $4.315_{-0.013}^{+0.018}$ & $4.355_{-0.014}^{+0.017}$ \\
\hline Average Surface Gravity, $\log \left(g_{\text {avg }}\right)(\mathrm{cgs})^{\mathrm{b}}$ & $4.207_{-0.022}^{+0.004}$ & $4.174_{-0.012}^{+0.019}$ & $4.164_{-0.009}^{+0.014}$ & $4.169_{-0.014}^{+0.011}$ \\
\hline Equatorial Surface Gravity, $\log \left(g_{\mathrm{e}}\right)(\mathrm{cgs})$ & $3.813_{-0.041}^{+0.091}$ & $3.968_{-0.025}^{+0.028}$ & $3.848_{-0.054}^{+0.032}$ & $3.593_{-0.082}^{+0.054}$ \\
\hline$v \sin i\left(\mathrm{~km} \mathrm{~s}^{-1}\right)$ & $157.4_{-44.9}^{+5.8}$ & $142.2_{-21.1}^{+13.1}$ & $146.2_{-16.2}^{+11.0}$ & $164.7_{-11.5}^{+13.4}$ \\
\hline Total Luminosity, $L_{\text {tot }}\left(L_{\odot}\right)$ & $55.21_{-3.14}^{+5.67}$ & $62.60_{-2.23}^{+9.83}$ & $58.35_{-3.08}^{+6.26}$ & $53.50_{-5.37}^{+1.73}$ \\
\hline Apparent Luminosity, $L_{\text {app }}\left(L_{\odot}\right)$ & $71.17_{-3.99}^{+3.72}$ & $72.01_{-1.50}^{+11.17}$ & $72.49_{-2.04}^{+7.67}$ & $72.99_{-7.22}^{+1.24}$ \\
\hline Visibility $\chi^{2}$ & 12.99 & 13.23 & 13.01 & 12.85 \\
\hline Photometry $\chi^{2}$ & 9.68 & 8.92 & 8.74 & 8.75 \\
\hline Total $\chi^{2}$ & 22.67 & 22.15 & 21.75 & 21.60 \\
\hline
\end{tabular}

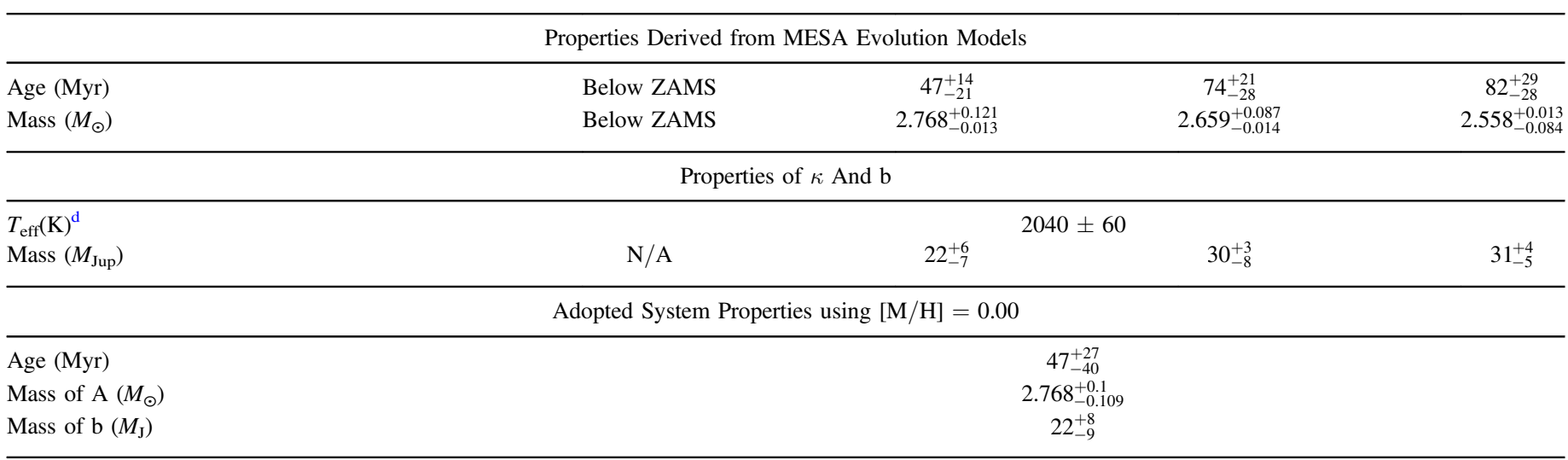

Notes.

${ }^{\mathrm{a}}$ We adopt as our final results those from the solar metallicity models.

${ }^{b}$ The average quantities presented here are averaged across the entire surface of the model star.

${ }^{c}$ The average angular diameter is determined using the average radius and the distance.

d From H13.

\section{RESULTS AND DISCUSSION}

\subsection{The Properties of $\kappa$ And $A$}

We use the model discussed in Section 3 to determine the age of $\kappa$ And $\mathrm{A}$ for four different internal metallicities $([\mathrm{M} / \mathrm{H}]$ $=+0.14,0.0,-0.14$, and -0.28$)$ corresponding to the $+1-, 0-$, $-1-$, and $-2 \sigma$ uncertainties in $[\mathrm{M} / \mathrm{H}]$, respectively. For the solar metallicity model, we find a radius for the host star ranging from $2.303_{-0.016}^{+0.039} R_{\odot}$ at the equator to $1.959_{-0.028}^{+0.033} R_{\odot}$ at the pole with an average of $2.109_{-0.018}^{+0.032} R_{\odot}$. This oblateness is, in part, due to an equatorial velocity of $283.8_{-16.1}^{+13.4} \mathrm{~km} \mathrm{~s}^{-1}$, which corresponds to an angular rotation rate relative to the critical rate, $\omega$, of $0.854_{-0.028}^{+0.021}$ and which with the modeled inclination of $30^{\circ} 1_{-4.8}^{+3.1}$ gives a modeled $v \sin i$ of $142.2_{-21.1}^{+13.1} \mathrm{~km} \mathrm{~s}^{-1}$. Our modeled effective temperature ranges from $12050_{-39}^{+448} \mathrm{~K}$ at the pole to $10342_{-138}^{+384} \mathrm{~K}$ at the equator with an average of $11327_{-44}^{+421} \mathrm{~K}$, and together with the modeled radius profile, yield a total luminosity of $62.60_{-2.23}^{+9.83} L_{\odot}$ and apparent luminosity of $72.01_{-1.50}^{+11.17} L_{\odot}$. We model an average 


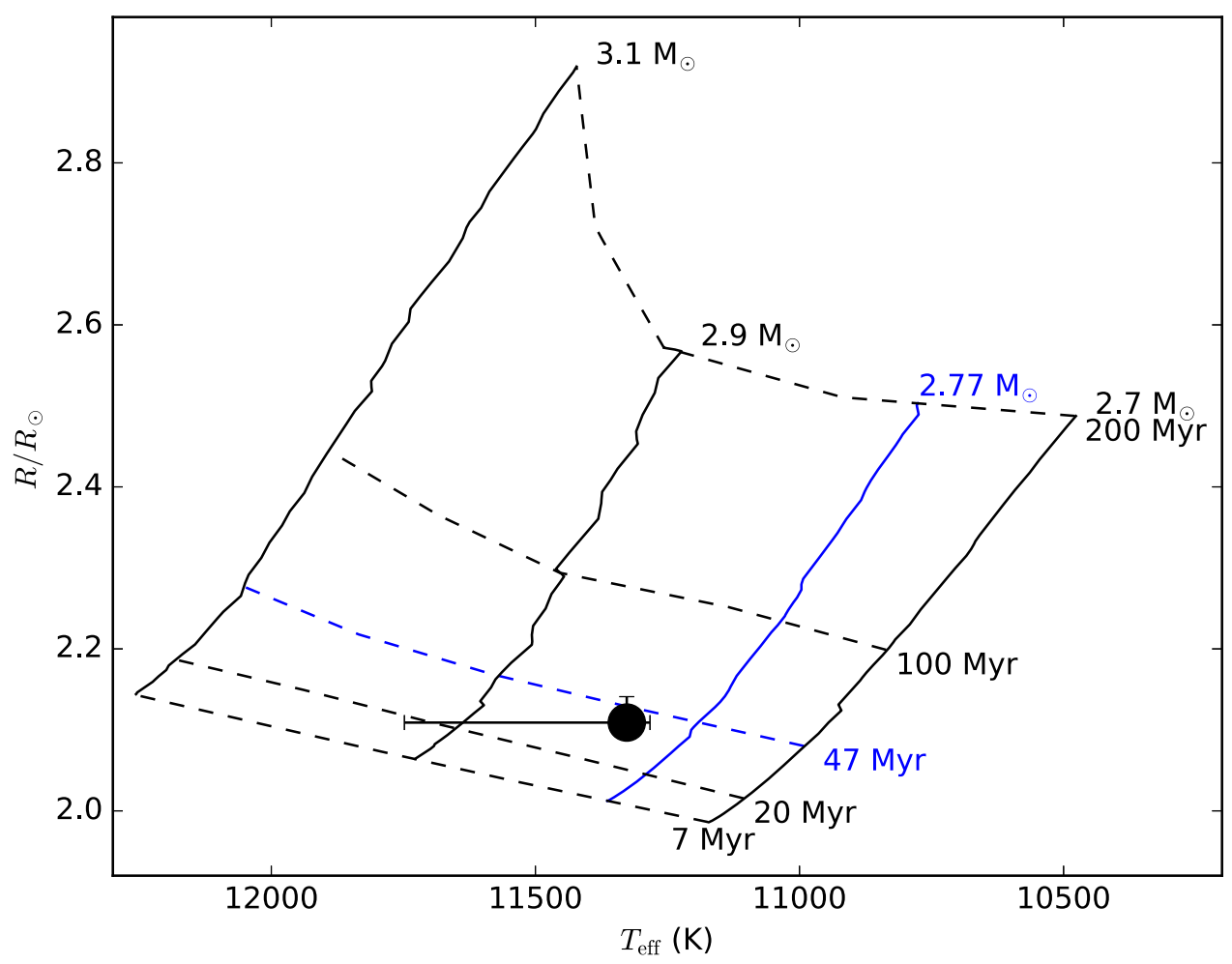

Figure 2. Solid lines show the evolution in radius and effective temperature according to the mass tracks of the MESA evolution models for masses ranging from 2.7 to $3.1 M_{\odot}$. The dashed lines are isochrones showing the radius and effective temperatures of stars with this range of masses at ages ranging from 7 to 200 Myr. Both the mass tracks and isochrones were calculated for solar metallicity and interpolated to the modeled rotation velocity of the star.

surface gravity $\left(\log \left(g_{\text {avg }}\right)\right)$ of $4.174_{-0.012}^{+0.019}$ dex, which is only slightly larger than previous measurements of the star's $\log (g)$ ranging from 3.8 to 4.1 dex (Fitzpatrick \& Massa 2005; Wu et al. 2011; Bonnefoy et al. 2014).

The age and mass we determine using the best-fitting model with a solar metallicity are $47_{-21}^{+14} \mathrm{Myr}$ and $2.768_{-0.013}^{+0.121} M_{\odot}$, respectively. This young age is due, in large part, to the low inclination $\left(\sim 30^{\circ}\right)$ and large rotation velocity $(\sim 85 \%$ critical), which implies that the apparent luminosity is brighter than the total luminosity because of the effects of gravity darkening and which also changes where the zero-age main sequence (ZAMS) lies on the HR diagram.

Most of our modeled parameters show broad agreement between the four different internal metallicities tested; however, the age and the mass show a significant correlation with metallicity (e.g., a lower metallicity corresponds to an older age and a lower mass). Given how strongly the internal metallicity affects the modeled mass and age of the host star, we adopt the ages and masses determined at the $1 \sigma$ uncertainties in the metallicity as the bounds to our final uncertainties in the age and mass. The supersolar metallicity model $([\mathrm{M} / \mathrm{H}]=+0.14)$ has a radius and luminosity below the ZAMS, so we adopt the age of the ZAMS, $\sim 7 \mathrm{Myr}$, as the lower bound of the uncertainty in the age. Given the trend of decreasing mass of $\sim 0.1 M_{\odot}$ for every $1 \sigma$ decrease in metallicity, we adopt an upper bound of the uncertainty in our mass to be $0.1 M_{\odot}$ Thus, our final estimate of the age and mass of $\kappa$ And $\mathrm{A}$ is $47_{-40}^{+27} \mathrm{Myr}$ and $2.768_{-0.109}^{+0.1} M_{\odot}$, respectively.

We note that a more recent age estimate of the Columba association by Bell et al. (2015) finds it to be $42_{-4}^{+6} \mathrm{Myr}$, which is in excellent agreement with our age estimate for $\kappa$ And A. Despite its outlying Galactic $\mathrm{Y}$ position with respect to
Columba $(2.7 \sigma ; \mathrm{H} 13)$, the agreement in age suggests that its kinematic association with young nearby groups should be reconsidered.

\subsection{A Comparison to Previous Age Estimates}

H13 use a variety of methods to estimate the age of $\kappa$ And A, finding ages ranging from $\sim 50$ to $400 \mathrm{Myr}$. Their adopted age of $220 \pm 100 \mathrm{Myr}$ is based on a comparison between the predictions of the Geneva evolution models (Ekström et al. 2012) that account for a rotation rate of $\omega=0.4$ and the $\log (g) \quad(4.10 \mathrm{dex})$ and $T_{\text {eff }}(11,366 \mathrm{~K})$ measured by Fitzpatrick \& Massa (2005). This age estimate is significantly older than both the traditionally adopted age of the Columba association $\left(30 \mathrm{Myr}\right.$ ) and our estimate $\left(47_{-40}^{+27} \mathrm{Myr}\right)$. H13 do note that such a young age is possible if the host star is rapidly rotating $\left(V_{\mathrm{e}} / V_{\text {crit }} \simeq 0.95\right)$ with an very low orientation $\left(\simeq 22^{\circ}\right)$, which is what we have found with this work.

DH15 use Strömgren photometry of Hauck \& Mermilliod (1997) to determine a $\log (g)$ of $4.35 \pm 0.14 \mathrm{dex}$ and $T_{\text {eff }}$ of $11903 \pm 405 \mathrm{~K}$. From this, they interpolate between the isochrones generated by the evolution models of Bressan et al. (2012) and Ekström et al. (2012) to estimate an age of $16 \mathrm{Myr}$. Superseding this interpolated estimate, they use a more thorough Bayesian approach and find a 95\% confidence interval of 29-237 Myr with a median age of $150 \mathrm{Myr}$.

In an attempt to determine how much the choice of evolution model affects the estimated age, we compare the $\log (g)$ and $T_{\text {eff }}$ values used by both $\mathrm{H} 13$ and DH15 to the MESA evolution models used here. We estimate an age of $185 \mathrm{Myr}$ and $13 \mathrm{Myr}$ using the $\log (g)$ and $T_{\text {eff }}$ values used by $\mathrm{H} 13$ and DH15, respectively. These estimates are lower than the estimates made 


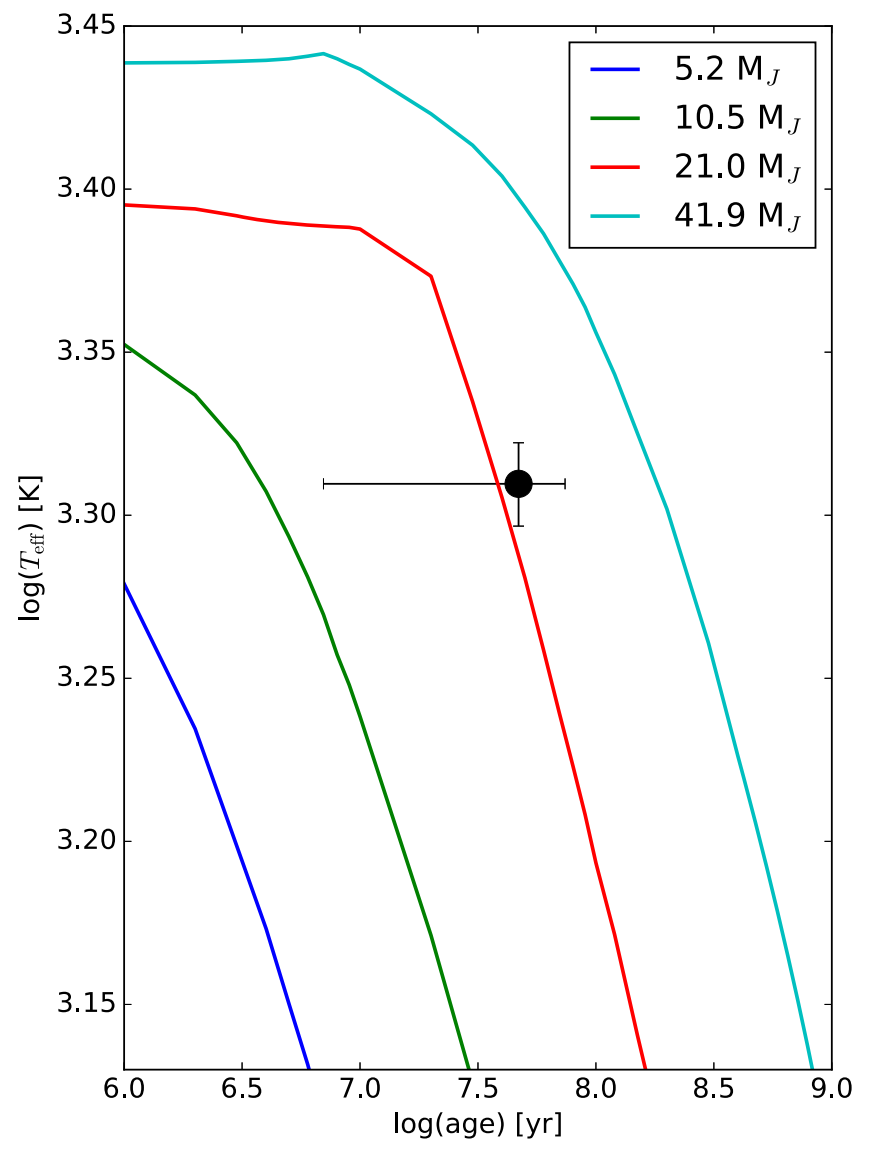

Figure 3. Solid lines show how the BHAC 15 evolution models predict substellar objects cool over time for masses ranging from 5.2 to $41.9 M_{\mathrm{J}}$. The black point shows the effective temperature of $\kappa$ And b $(2040 \pm 60 \mathrm{~K}$; H13) and its age $\left(47_{-40}^{+27} \mathrm{Myr}\right.$; this work).

by these two studies by $\sim 20 \%$, which is smaller than the uncertainties in the age estimates.

\subsection{The Mass of $\kappa$ And $b$}

In order to determine the mass of $\kappa$ And $b$, we compare our age estimate for the host star and the spectroscopically determined effective temperature of the companion $(2040 \pm 60 \mathrm{~K} ; \mathrm{H} 13)$ to the predictions of the updated BHAC15 models of Baraffe et al. (2015). Uncertainties in the companion mass are determined by using this method to calculate the mass corresponding to the four points representing the $1 \sigma$ uncertainties in the age and effective temperature of the companion. With this technique, we find a mass of $22_{-9}^{+8} M_{\mathrm{J}}$ with the uncertainties dominated by the uncertainty in the age which is dominated by the uncertainty in the metallicity. Figure 3 shows the effective temperature of $\kappa$ And b from $\mathrm{H} 13$ and our final estimate for the age of the system along with the cooling tracks of the BHAC15 models.

\section{SUMMARY}

We present new PAVO/CHARA interferometric observations of $\kappa$ And A. Using these observations, the star's photometry, and its $v$ sin $i$, we constrain an oblate star model from which we calculate various fundamental parameters.
These parameters include the star's luminosity, radius profile, and equatorial rotation velocity that are compared to the predictions of the MESA evolution models in order to estimate an age and mass for the star. Four internal metallicities $([\mathrm{M} / \mathrm{H}]=+0.14,0.0,-0.14$, and -0.28$)$ are tested, and we find that metal-rich models yield higher-mass and younger-age more metal-poor models.

Because the internal metallicity of the star is expected to be solar $([\mathrm{M} / \mathrm{H}]=0.00 \pm 0.14)$, we adopt the solar metallicity model with the uncertainties in our final age and mass governed by the uncertainty in the metallicity. With this model, we determine an age of $47_{-40}^{+27} \mathrm{Myr}$ for the system and a mass of $2.768_{-0.109}^{+0.1} M_{\odot}$ for $\kappa$ And A. Based on this age, the effective temperature of the companion, and the BHAC15 evolution models, we determine a mass of $\kappa$ And b of $22_{-9}^{+8} M_{\text {Jup. }}$.

J.J. thanks Dr. Sasha Hinkley and Dr. Josh Schlieder for their helpful comments. This work is based upon observations obtained with the Georgia State University Center for High Angular Resolution Astronomy Array at Mount Wilson Observatory. The CHARA Array is supported by the National Science Foundation under grants AST-1211929 and AST1411654. Institutional support has been provided from the GSU College of Arts and Sciences and the GSU Office of the Vice President for Research and Economic Development. J.J. and R.J.W. acknowledge support from NSF AAG grants 1009643 and 1517762.

\section{REFERENCES}

Baraffe, I., Chabrier, G., Barman, T. S., Allard, F., \& Hauschildt, P. H. 2003, A\&A, 402, 701

Baraffe, I., Homeier, D., Allard, F., \& Chabrier, G. 2015, A\&A, 577, A42 Bell, C. P. M., Mamajek, E. E., \& Naylor, T. 2015, MNRAS, 454, 593 Boden, A. F. 2007, NewAR, 51, 617

Bodenheimer, P., D’Angelo, G., Lissauer, J. J., Fortney, J. J., \& Saumon, D. 2013, ApJ, 770, 120

Boggess, A., Carr, F. A., Evans, D. C., et al. 1978, Natur, 275, 372

Bonnefoy, M., Currie, T., Marleau, G.-D., et al. 2014, A\&A, 562, A111

Brandt, T. D., \& Huang, C. X. 2015, ApJ, 807, 58

Bressan, A., Marigo, P., Girardi, L., et al. 2012, MNRAS, 427, 127

Caffau, E., Ludwig, H.-G., Steffen, M., Freytag, B., \& Bonifacio, P. 2011, SoPh, 268, 255

Carson, J., Thalmann, C., Janson, M., et al. 2013, ApJL, 763, L32

Castelli, F., \& Kurucz, R. L. 2004, arXiv:astro-ph/0405087

Chen, L., Hou, J. L., \& Wang, J. J. 2003, AJ, 125, 1397

David, T. J., \& Hillenbrand, L. A. 2015, ApJ, 804, 146

Ekström, S., Georgy, C., Eggenberger, P., et al. 2012, A\&A, 537, A146

Espinosa Lara, F., \& Rieutord, M. 2011, A\&A, 533, A43

Fitzpatrick, E. L., \& Massa, D. 2005, AJ, 129, 1642

Glebocki, R., \& Gnacinski, P. 2005, yCat, 3244, 0

Hartman, J. D., Bakos, G. Á, Buchhave, L. A., et al. 2015, AJ, 150, 197

Hauck, B., \& Mermilliod, M. 1997, yCat, 2215, 0

Hinkley, S., Pueyo, L., Faherty, J. K., et al. 2013, ApJ, 779, 153

Husser, T.-O., Wende-von Berg, S., Dreizler, S., et al. 2013, A\&A, 553, A6

Ireland, M. J., Mérand, A., ten Brummelaar, T. A., et al. 2008, Proc. SPIE, 7013, 24

Jones, J., White, R. J., Boyajian, T., et al. 2015, ApJ, 813, 58

Mermilliod, J. C. 2006, yCat, 2168, 0

Mollière, P., \& Mordasini, C. 2012, A\&A, 547, A105

Paxton, B., Bildsten, L., Dotter, A., et al. 2011, ApJS, 192, 3

Paxton, B., Cantiello, M., Arras, P., et al. 2013, ApJS, 208, 4

Roche, E. 1873, Ann. de l'Acad. Sci. Montpellier, 8, 235

Roddier, F. 1981, Progress in Optics, Vol. 19 (Amsterdam: North-Holland)

Royer, F., Zorec, J., \& Gómez, A. E. 2007, A\&A, 463, 671

Selby, M. J., Hepburn, I., Blackwell, D. E., et al. 1988, A\&AS, 74, 127

Spiegel, D. S., Burrows, A., \& Milsom, J. A. 2011, ApJ, 727, 57 
Takeda, Y., \& Sadakane, K. 1997, PASJ, 49, 367

ten Brummelaar, T. A., McAlister, H. A., Ridgway, S. T., et al. 2005, ApJ, 628,453

Thompson, G. I., Nandy, K., Jamar, C., et al. 1978, Catalogue of Stellar Ultraviolet Fluxes. A Compilation of Absolute Stellar Fluxes Measured by the Sky Survey Telescope (S2/68) Aboard the ESRO Satellite TD-1 (Swindon: Science Research Council)

van Belle, G. T. 2012, A\&ARv, 20, 51

van Leeuwen, F. 2007, A\&A, 474, 653
Varenne, O., \& Monier, R. 1999, A\&A, 351, 247

von Zeipel, H. 1924a, MNRAS, 84, 665

von Zeipel, H. 1924b, MNRAS, 84, 684

Wesselius, P. R., van Duinen, R. J., de Jonge, A. R. W., et al. 1982, A\&AS, 49,427

Winn, J. N., \& Fabrycky, D. C. 2015, ARA\&A, 53, 409

Wu, Y., Singh, H. P., Prugniel, P., Gupta, R., \& Koleva, M. 2011, A\&A, 525, A71

Zuckerman, B., Rhee, J. H., Song, I., \& Bessell, M. S. 2011, ApJ, 732, 61 